ORIGINAL ARTICLE

\title{
Occupational magnetic field exposure among women in Stockholm County, Sweden
}

\author{
U M Forssén, G Mezei, G Nise, M Feychting
}

Occup Environ Med 2004;61:594-602. doi: 10.1136/oem.2003.009654

See end of article for authors' affiliations .....................

Correspondence to: Dr U Forssén, Institute of Environmental Medicine, Karolinska Institutet, PO Box 210, SE-171 77 Stockholm, Sweden Ulla.Forssen@imm.ki.se

Accepted 28 November 2003

\begin{abstract}
Background: Most epidemiological studies on adverse health effects among women in relation to occupational magnetic field exposure have been based on information about men's exposure.

Aims: To create a job-exposure matrix for occupational exposure to extremely low frequency magnetic fields among women.

Methods and Results: Measurements were performed using personal magnetic field meters (Emdex Lite) carried by the subjects for 24 hours on a normal workday. Subjects were volunteer women working in the occupations identified as common among women in Stockholm County based on the 1980 census. A total of 471 measurements were made in 49 different occupations, with a minimum of 5 and a maximum of 24 measurements in each occupation. The included occupations cover about $85 \%$ of the female population gainfully employed in 1980. Parameters representing average and peak magnetic field exposures, temporal change in the exposure, and proportion of time spent above certain exposure levels were calculated both for the workday and for the total 24 hour period grouped by occupational titles. The occupations with higher than average exposure were cashiers, working proprietors in retail trade, air stewardesses, dental nurses, cooks, post-office clerks and kitchen maids.

Conclusions: This new job-exposure matrix substantially increases the knowledge about magnetic field exposure among women and can be used for exposure assessment in future studies.
\end{abstract}

$\mathrm{E}$ pidemiological studies of the relation between occupational magnetic field exposure and adverse health effects have mainly focused on health effects among men. ${ }^{1}$ One reason is that many occupations that have higher than average levels of magnetic fields are industrial and electrical occupations, which are typically held by men. For outcomes among women the major research question has been whether there might be an association between magnetic field exposure and breast cancer. ${ }^{1}$ Another endpoint for women evaluated in relation to exposure to magnetic fields has been miscarriage. ${ }^{2} 3$ In studies on women the lack of occupational exposure information for women presents a problem since there is an obvious possibility of exposure misclassification if the exposure assessment is based on information for men. Also, because few women work in the highly exposed occupations, the statistical power in studies on women tends to be low.

Two studies have previously been performed with the aim of providing information about exposure to extremely low frequency $(50 \mathrm{~Hz})$ magnetic fields in occupations that are common among women. ${ }^{45}$ However, one includes only theoretical assignments of exposure to different occupations and the other does not cover a wide range of occupations. We performed our study to provide a stronger basis for improved occupational magnetic field exposure assessment among women in future epidemiological studies. The primary goal was to create a job-exposure matrix (JEM) for women by doing personal measurements in the most common occupations among women in Stockholm County. We also compared occupational exposure levels of magnetic fields between men and women and evaluated the magnitude of potential misclassification when men's exposure information was applied to women.

\section{METHODS}

From the 1980 Swedish Census we identified the most common occupations among women in Stockholm County.
We aimed to cover at least $80 \%$ of the women gainfully employed. We included the most frequent occupations and also some less common with potentially high exposure. Altogether we performed 471 measurements in 49 occupations. The number of measurements in each occupation ranged between 5 and 24 depending on how common the occupation was; more measurements were made in more common occupations. We also sought to distribute the measurements representatively among the subgroups of each occupation and additional measurements were made if the occupations had heterogeneous job tasks and work sites or if the preliminary results showed large variation between subjects. Workplaces conceivable as employers for the different occupations were identified through the Yellow Pages, the company phone book, and through home pages of major organisations such as Stockholm County Council and City of Stockholm. The types of workplaces chosen were reviewed by an occupational hygienist to ensure that they were representative for that particular occupation. We also tried to choose work sites so that the measurements would be spread geographically within Stockholm County. The occupations were coded according to the Nordic version of the International Standard Classification of Occupations (ISCO) that was used in the 1980 Swedish census.

Subjects were volunteer women working in one of the identified occupations in Stockholm County. When a work site had been identified, the manager was contacted; if they were willing to participate they were asked to identify one of the employees that held an occupation that would fit our description. We thereafter contacted that woman and asked if she wanted to participate. Both the manager and the participant received full information about the background and purpose of the study.

Abbreviations: JEM, job-exposure matrix; $\mu \mathrm{T}$, micro Tesla; $\mathrm{RC}$, rate of change; RCs, standardised rate of change; TWA, time weighted average; ISCO, international standard classification of occupations 


\section{Main messages}

- Assessments of occupational ELF magnetic field exposure in women have often been based on measurements in men, which has lead to loss of information and possibly misclassification of the exposure among women.

- Personal 24 hour magnetic field measurements were performed on 471 women covering the most common occupations; a job-exposure matrix was created with a number of different exposure parameters, making it available for exposure assessment in future studies on power frequency magnetic fields and health outcomes among women.

- Several new highly exposed occupations that are common among women were identified.

The measurements were performed with personal magnetic field meters (Emdex Lite, Enertech Consultants, Campbell, California) carried by the subjects on a belt by the hip for 24 hours. The meter measures magnetic fields with a resolution of $0.01 \mu \mathrm{T}$ in the range $40-1000 \mathrm{~Hz}$ and was calibrated by the manufacturer at $50 \mathrm{~Hz}$ with $\pm 2 \%$ accuracy. The calibration within and between all meters that were used in the study (a total of 11 meters) was checked at $50 \mathrm{~Hz}$ with a Helmholtz coil magnetic field generator located at the Swedish Radiation Protection Authority, and the functionality of the meters was controlled continuously during the study. The magnetic field was measured and stored in the memory of the meter every 4th second, and the mode was set to not displaying the measured field to discourage the subjects to test certain environments for high fields.

At the day of the measurement an assistant from the research team delivered the meter and gave detailed instructions, both oral and written, about how to handle the meter. The subjects were instructed to carry the meter at all times possible and if they had to take it off record that time in the diary. They were instructed to place the meter beside the pillow or on a bedside table during sleep and to be careful not to place the meter close to any electrical appliances, such as clock radios. In a short questionnaire the subjects were asked to describe their job tasks and to report whether the day of the measurement was in any way different from a normal workday. During the measurement the subjects also kept a diary of their whereabouts from which we could identify time spent at work, at home, and elsewhere. After 24 hours the assistant picked up the meter and brought it back to the Karolinska Institute where the data were downloaded to a PC. The data from the diary were also entered and the results were thoroughly scrutinised; suspicious data were followed up by an immediate phone call to the subject. In cases of invalid data (for example, the subject had taken the meter off for some reason) that was discovered either through the follow up or directly in the diary, the data for that period were excluded. Measurements with odd appearance without any explanation were included with no further action. A plot showing the results was sent to each participant.

All subjects gave their consent to participation and the study was approved by the ethical committee at Karolinska Institutet (Dnr: 00-432).

\section{Analyses}

For each subject we calculated a number of different parameters describing the exposure: time weighted average

\section{Policy implications}

- There is a need for gender specific exposure assessment in studies of occupational ELF magnetic field exposure.

(TWA), median, 90th centile, maximum value, the proportion of time spent at different levels of exposure $(<0.1 \mu \mathrm{T}, 0.1-$ $0.19 \mu \mathrm{T}, 0.2-0.29 \mu \mathrm{T}$, and $\geqslant 0.3 \mu \mathrm{T}$ ), and finally the rate of change (RC) and the standardised rate of change (RCs). The $\mathrm{RC}$ is a measure of both variability and first lag autocorrelation between successive measurements. The RCs is the RC divided by the standard deviation for the measurement period and is hence an estimate of the first lag autocorrelation only. ${ }^{6}$ All parameters were calculated both for the workday only and for the total 24 hour period. To summarise over occupations we calculated the arithmetic mean, the geometric mean, and the median for each occupation. We used the standard deviation and the 1st and 3rd quartile to describe the variation between subjects within an occupation.

We defined five work site environments and used the knowledge we had about the employer in combination with descriptions of job tasks and the diary to categorise as many subjects as possible according to these. The environments were "health care", "schools and childcare", "large scale kitchens", "offices", and "shops and stores". Health care was divided into "hospitals" and "elsewhere", where elsewhere includes private clinics and primary health care centres. There were 316 subjects in the study (67\%) who fitted into one of these environments.

Correlation between different parameters was estimated using both Pearson's and Spearman's correlation coefficients and was based on the subjects' individual estimates. Finally, we linked the JEM estimates to the 1980 census and calculated the exposure distribution for a large sample of women gainfully employed in Stockholm County in 1980 $(n=163336)$. To make an evaluation of the new information provided by this JEM for women we also linked the estimates from another JEM for men, developed by Floderus and colleagues, ${ }^{7}$ to the gainfully employed women in the 1980 census. The JEM by Floderus et al has been used in several previous studies as occupational exposure assessment for women. ${ }^{8-11}$ For women with occupations that had exposure information in both JEMs we calculated sensitivity and specificity. Sensitivity was defined as the proportion of the truly exposed ( $\geqslant 0.20 \mu \mathrm{T}$ as determined by the women's JEM) that was classified as exposed by the men's JEM. Specificity was defined as the proportion of truly unexposed $(<0.12 \mu \mathrm{T}$ as determined by the women's JEM) that was classified as unexposed by the men's JEM. ${ }^{12}$

\section{RESULTS}

The measurements were performed between March 2001 and October 2002 and of the 486 women who agreed to participate, 471 were included in the analyses. The reasons for exclusion of subjects were less than four hours at work $(\mathrm{n}=2)$, wrong occupation $(\mathrm{n}=5)$, or the job site located very close to a power line $(n=4)$. For 23 subjects the first measurement failed because of technical problems; 19 of them agreed to do the measurement again and four refused and were therefore excluded. The age of the subjects ranged from 20 to 66 years with an average of 43 years, and the average time spent at work was 8.3 (range 4-22) hours. The arithmetic mean across all subjects' TWA was $0.20 \mu \mathrm{T}$, the median of the TWA was $0.15 \mu \mathrm{T}$, the 1st quartile $0.09 \mu \mathrm{T}$, and the 3rd quartile $0.25 \mu \mathrm{T}$. 


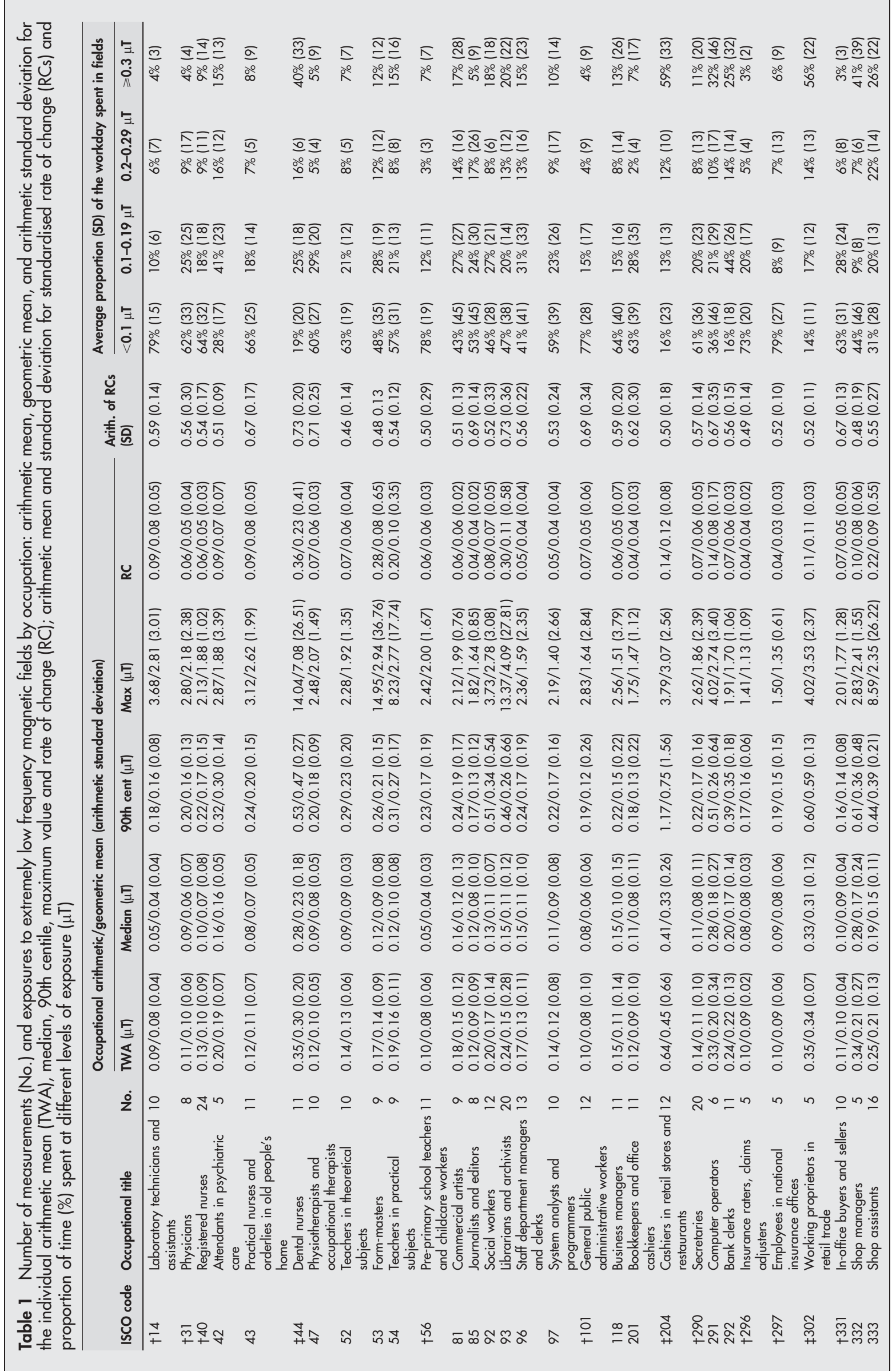




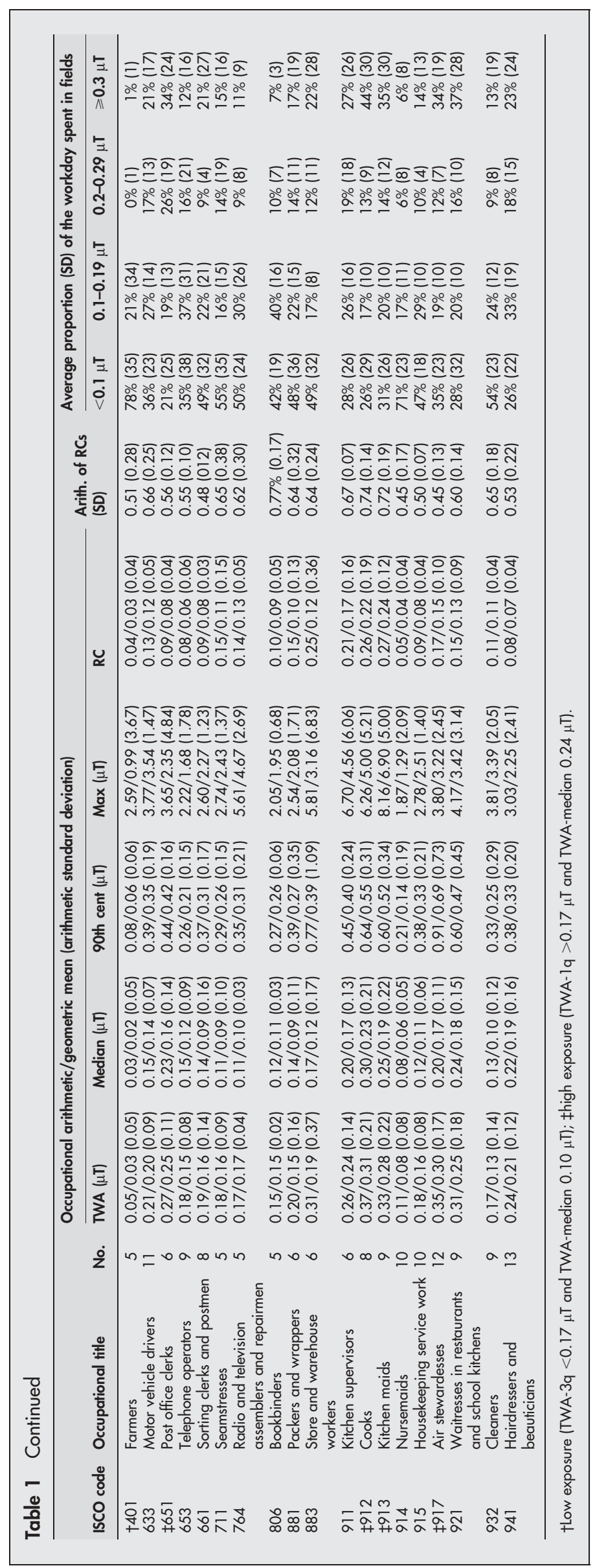


Table 1 summarises the occupations by arithmetic mean, geometric mean, and standard deviation for a number of different parameters. For librarians and archivists (ISCO code 093), the occupational means are high because the subgroup librarian assistants have high fields. They had an arithmetic mean of $0.41 \mu \mathrm{T}$ and a geometric mean of $0.29 \mu \mathrm{T}$, based on eight measurements. For air stewardesses (ISCO code 917) we did measurements on both short and long distance (transatlantic) flights. They were all highly exposed; for short distance flights arithmetic and geometric means were 0.38 and $0.33 \mu \mathrm{T}$ and for long distance flights they were 0.28 and $0.26 \mu \mathrm{T}$, respectively. To increase the discrepancy between high and low exposure we introduced a criterion based on the individual estimate of TWA. We defined occupations as having low exposure if the 3rd quartile of the TWA estimates was lower than $0.17 \mu \mathrm{T}$ and the median $0.10 \mu \mathrm{T}$ or less (these occupations are marked $\dagger$ in table 1$)$. Occupations with a median of $0.24 \mu \mathrm{T}$ or more and the lst quartile higher than $0.17 \mu \mathrm{T}$, was defined as highly exposed (marked $\ddagger$ in table 1 ). Using these criteria, the low exposure occupations covered about $29 \%$ of the gainfully employed women in Stockholm County 1980 and the highly exposed covered about 7\%.

Figure 1 illustrates the variation of the exposure levels within occupations and the overlap between different occupations. To improve the scaling two extreme values were omitted from fig 1A ( $1.4 \mu \mathrm{T}$ in ISCO code 093 and $2.58 \mu \mathrm{T}$ in ISCO code 204) and one from fig $1 \mathrm{~B}$ ( $1.35 \mu \mathrm{T}$ in ISCO code 043). To evaluate the impact of the time spent at home and elsewhere on the occupational estimates we replicated fig 1A, using TWA for all 24 hours on the individual level. Notice that all figs $1 \mathrm{~A}, 1 \mathrm{~B}, 2 \mathrm{~A}$, and $2 \mathrm{~B}$, are sorted according to the medians found for TWA exposure at work in fig 1A. When including all 24 hours, the exposure level in the highly exposed occupations decreased but was still about $0.20 \mu \mathrm{T}$. For the low exposure occupations the median increased slightly but remained around $0.10 \mu \mathrm{T}$. The strong increase for insurance raters (ISCO code 296) was due to one woman who had extremely high exposure at night.

Figure 2 shows the average (arithmetic) proportion of time spent at different levels of exposure, for the workday only (fig 2A) and for the total 24 hours (fig 2B). The two intermediate levels $(0.1-0.19 \mu \mathrm{T}$ and $0.2-0.29 \mu \mathrm{T})$ are presented with the same colouring. The occupations that were categorised as highly exposed according to the median in fig $1 \mathrm{~A}$, also spent most time (about $40 \%$ ) in fields $\geqslant 0.30$ $\mu \mathrm{T}$, whereas the low exposure group spent only about $5 \%$ of their time in fields $\geqslant 0.30 \mu \mathrm{T}$. For the entire 24 hour period (fig $2 \mathrm{~B}$ ), the proportion of time in high fields was decreased for occupations with a median over $0.20 \mu \mathrm{T}$ (the far right hand of the figure).

Table 2 presents the results for the work site environments. "Large scale kitchens" and "shops and stores" are both environments with high exposure. Table 3 shows the correlation between different parameters based on the individual measurements. Both median and geometric mean correlated well with arithmetic mean (TWA), and median to geometric mean showed almost full correspondence. The maximum value correlated with the rate of change but not with the 90th centile or any of the other parameters.

Table 4 shows the exposure distribution among women gainfully employed in Stockholm County in 1980. The results are presented separately for the two different JEMs (ours for women and Floderus et al for men) according to different cutoff points of the geometric mean of the TWA. For the occupations that had estimates in both JEMs the sensitivity was 0.3 and the specificity 0.6 , using JEM for women as gold standard. Few subjects who, according to the gold standard, had truly low exposure $(<0.12 \mu \mathrm{T})$ were categorised as highly exposed $(\geqslant 0.20 \mu \mathrm{T})$ by the JEM for men, but a large proportion with truly high exposure were categorised as having low exposure. For the intermediate levels the misclassification was substantial in all directions.

\section{DISCUSSION}

In this study we created a job-exposure matrix based on systematically performed magnetic field measurements in the 49 most common occupations among women in Stockholm County. We identified a group of occupations in which workers are exposed to higher than average magnetic field levels and spend a larger than average proportion of their time in high fields. Individual occupations showed large variation and overlap in measured magnetic field values. When exposure during 24 hours was taken into consideration the contrast between high and low exposure occupations was diluted but still discernible. Among the highest exposed were women working in kitchens and in shops, which comprises occupations that have not previously been identified as highly exposed to magnetic fields.

The main limitation of the study is the limited number of measurements in each occupation and that the subjects were all volunteers and thus not a random sample. To ensure representation the measurements were distributed within the occupations in collaboration with an occupational hygienist and the occupations with the largest variety also received the largest number of measurements. In future studies the measurements are recommended to focus on the occupations identified as highly exposed since they showed larger withinoccupation variability. Another shortcoming in this study is that the measurement was performed only at one occasion and the variability may be large between workdays or seasons. To address the day-to-day and seasonal variations by repeated measurements on the same subjects is difficult since a very large number of measurements would be necessary. Spreading the measurements between different individuals probably increases the general knowledge better. Finally, the meters used in this study were only able to record the magnetic fields every 4th second, leaving some time unrecorded where the fields could have varied unnoticed. This would especially have an impact on the rate of change metrics.

It is still unknown what aspect of magnetic field exposure might be aetiologically relevant for a possible cancer risk. Most epidemiological studies have used some exposure metric based on TWA. Other alternatives that have been suggested are time spent in high fields ${ }^{13}$ and short term variations in flux density. ${ }^{6}$ In this study we included estimates for both these parameters (proportion of time and rate of change) for the different occupations. Although the rate of change (RC) did not correlate very well with TWA on the individual level, most of the occupations with the highest level of RC were the same as the occupations with high TWA, but there were some exceptions.

The importance of distinguishing between different environments and job tasks within an occupation has been described in a recent report ${ }^{14}$ suggesting that combining job title with environment would improve the exposure precision. In our study too few of the occupations had more than one type of environment for further analyses. Instead we did a grouping of occupations to describe the exposure in different working environments. Our study showed low levels of exposure in occupations that are found in hospitals, schools, and offices. These findings are supported by two recent studies of occupational magnetic field exposure among women performed in the USA ${ }^{4}$ and in Canada. ${ }^{5}$ Both studies found low levels in schools and offices, but the Canadian study by Deadman and Infante-Rivard ${ }^{5}$ found high levels among nurses. However, no actual measurements were performed in that study. The study by McCurdy and 


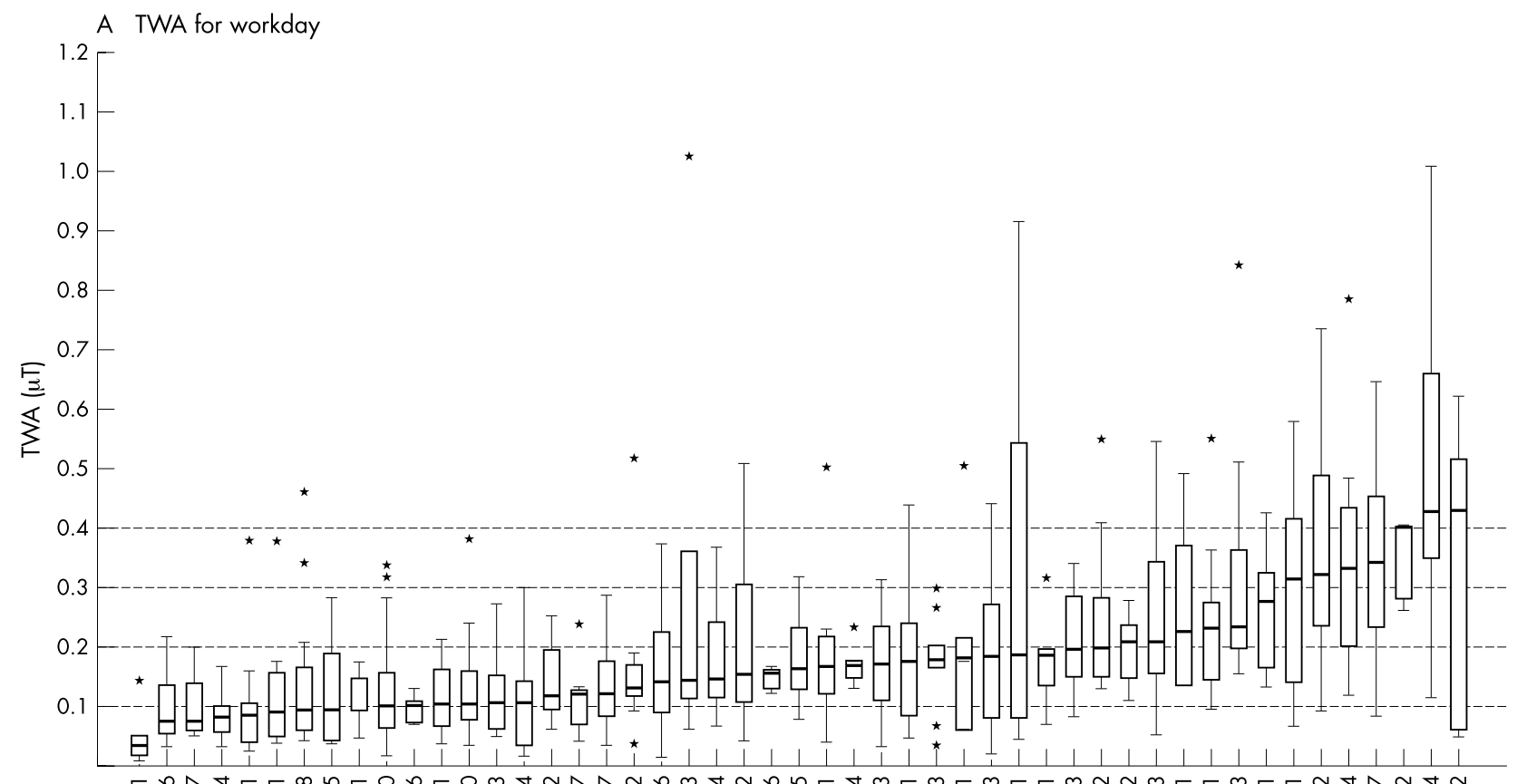

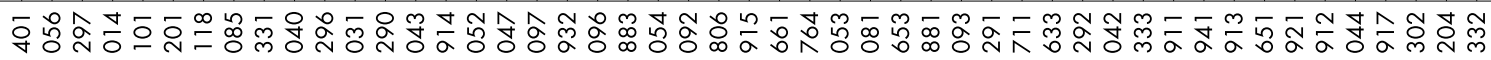
ISCO code

B TWA for 24 hours

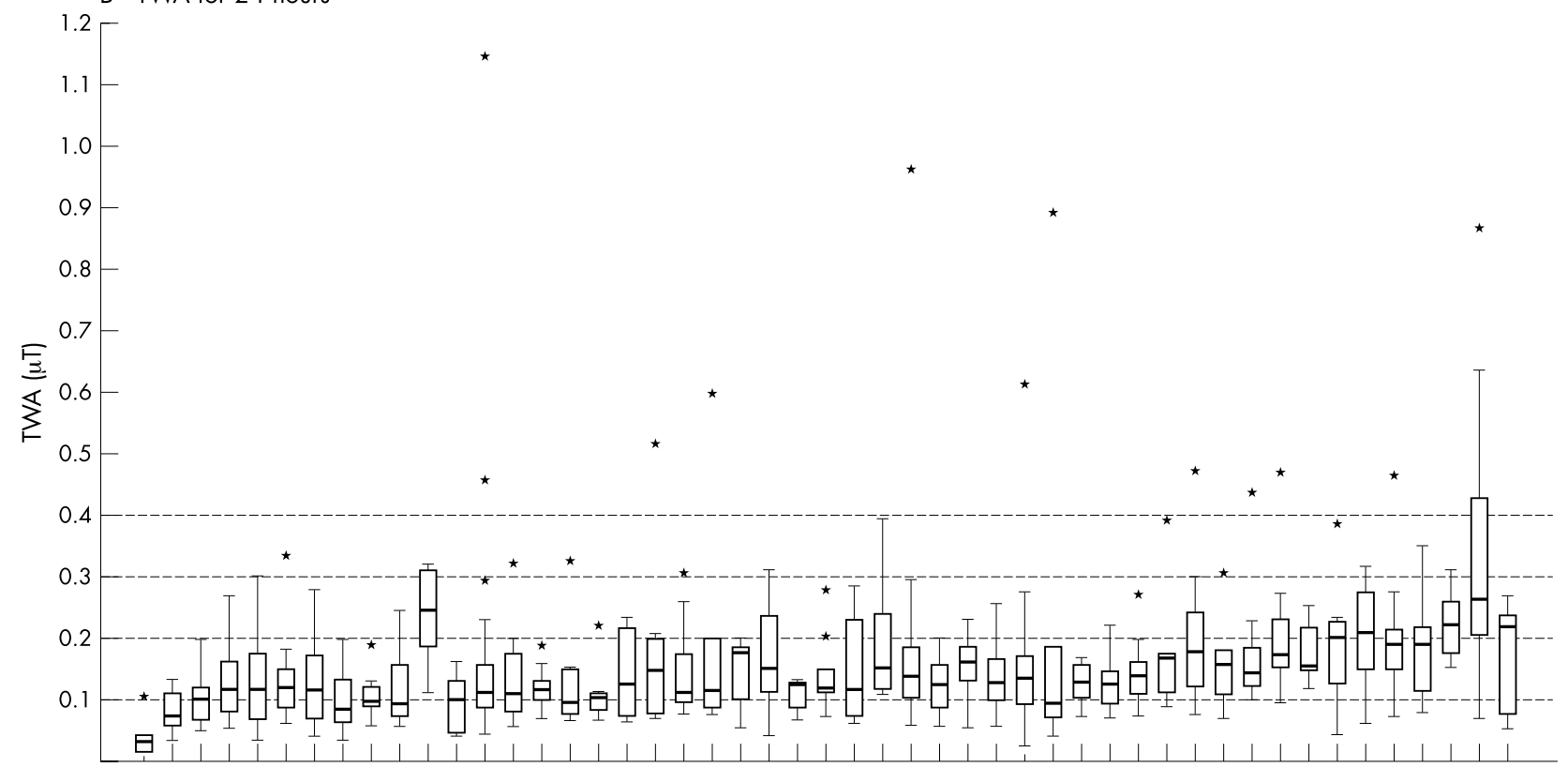

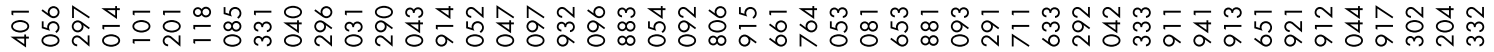
ISCO code

Figure 1 The time weighted average (TWA) of each individual grouped into occupations and sorted by the median in (A). The TWA is based on two different time periods: (A) workday only; (B) all 24 hours. For each occupation the box cover the interquartile range ( 1 st to 3rd quartile); the horizontal line across the box indicates the median, and the whiskers (vertical lines from the upper and lower edge of the box) cover the total range with exceptions of extreme values.

colleagues $^{4}$ is based on measurements in a sample of women within an ongoing breast cancer study and reported low levels among nurses.

Higher than average exposure in our study was found for air-stewardesses and in large scale kitchens, shops and stores, and libraries. Much of the exposure for air-stewardesses was experienced on board the aircraft, particularly at take off and landing. The aircraft use an electrical system that operates at $400 \mathrm{~Hz}$. In libraries the high levels were specific for librarian assistants. They work at the help desk close to the electronic scanner and other electric appliances that generate high fields. Educated librarians have different work tasks that in our study showed lower estimates of magnetic fields. The high levels among women working in kitchens 

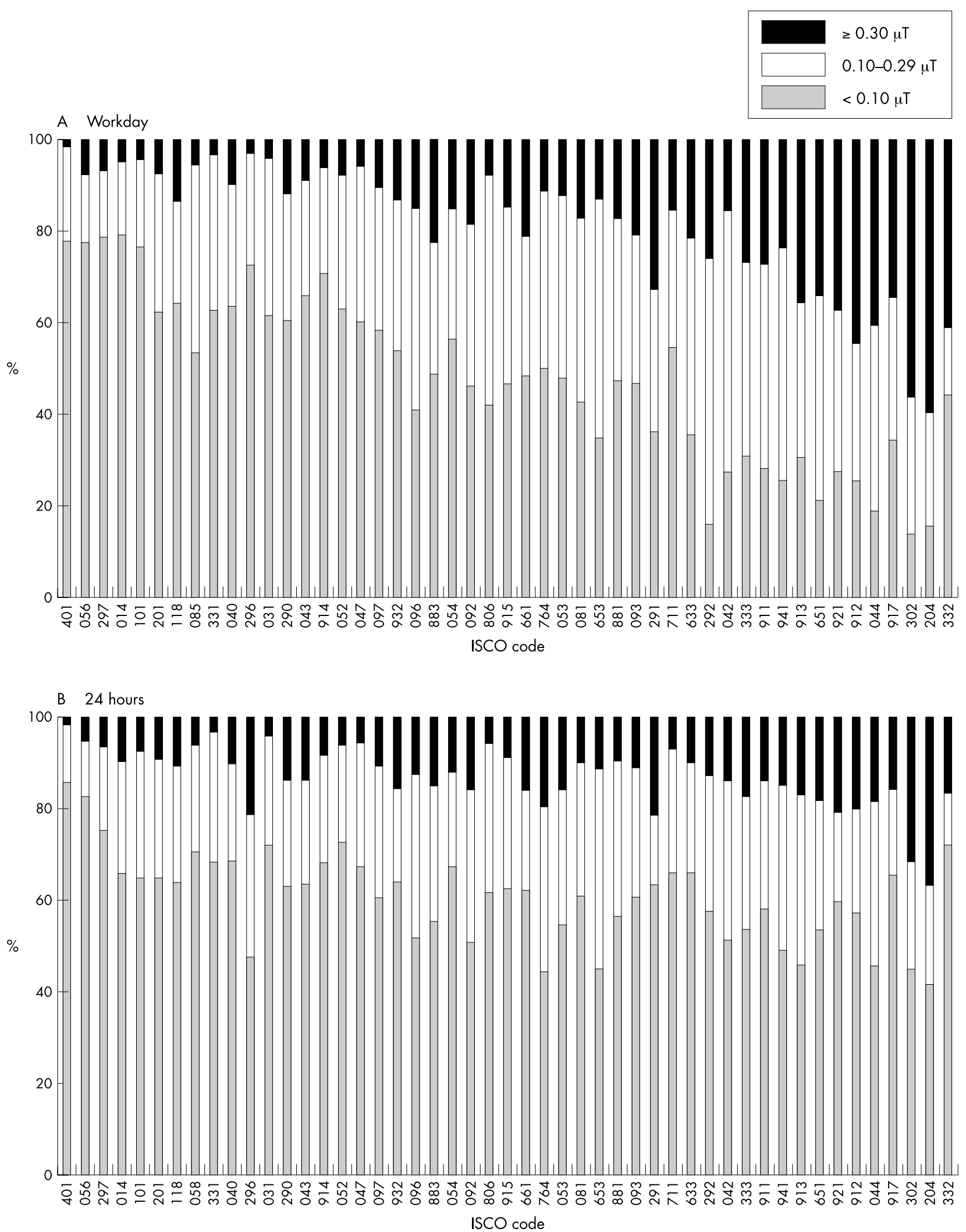

Figure 2 The average proportion (\%) of time spent at different levels ( $\mu$ T) of exposure, according to occupational (ISCO) code. The time is based on two different periods: (A) workday only; (B) all 24 hours.

were consistent over all occupations in these work sites but the variability between individuals was large. The two previous studies on women ${ }^{45}$ likewise reported high levels of magnetic field exposure in kitchens. Shops and stores have not previously been reported on specifically, but in our study they were shown to have among the highest levels of all occupations. One source of exposure could be the electronic article surveillance (EAS) systems. There are several different 


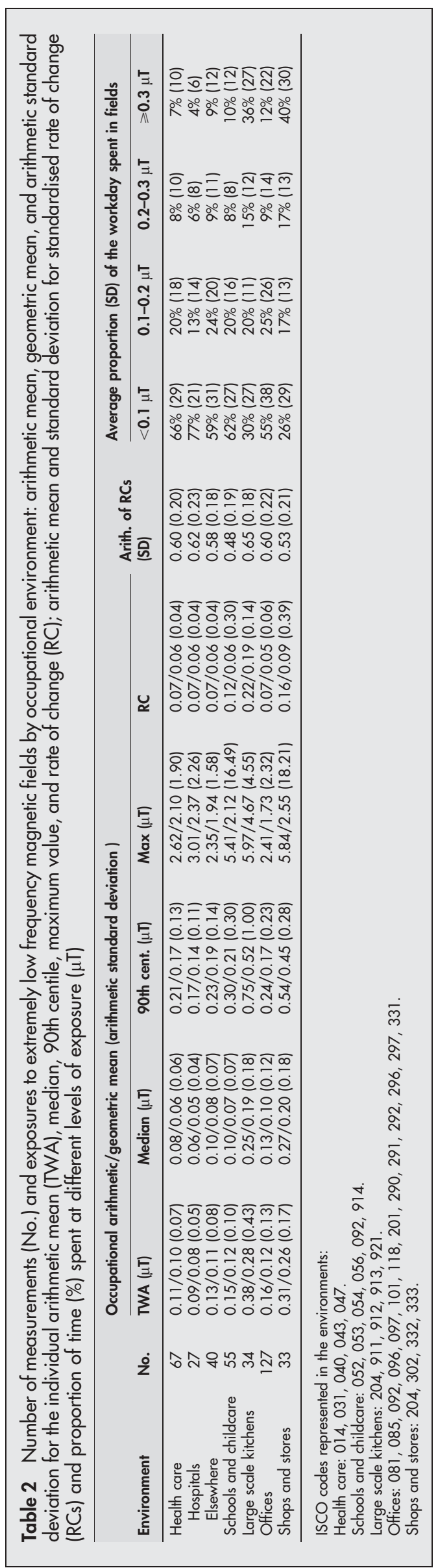

EAS systems on the market but only one operates at extremely low frequency and could be captured by the Emdex Lite. ${ }^{15}$ The Emdex Lite meter captures fields with frequencies up to $1000 \mathrm{~Hz}$ but the frequency could not be specified with this meter. Outside this range the fields are filtered out, but, although with significant attenuation, very strong fields might still be captured by the meter. If there are very strong fields of high frequencies in some occupations and the higher frequency is a risk factor by itself, this could cause confounding in epidemiological studies on extremely low frequency.

Of the 49 occupations in this study 29 were also covered by the study by Floderus and colleagues. ${ }^{7}$ That JEM was based on measurements on men and covers 100 different occupations; one of our goals was to make a comparison between occupational magnetic field exposure for men and women using this JEM. In general there was a good agreement between most of the occupations common to our JEM and the Floderus JEM, but for a number of occupations there were clear differences in the results. Differences in magnetic field exposure levels between men and women for packers (ISCO code 881), sorting clerks and postmen (ISCO code 661), and farmers (ISCO code 401) are almost certainly due to differences in tasks between men and women. The discrepancy for journalists (ISCO code 085) and commercial artists (ISC0 code 081) depends to a large extent on use of equipment that could differ between the sexes but could also be explained by the difference in time between the studies. The Floderus measurements were performed in 1989-91; our measurements were performed in 2001-02. The reason for the different results for bookkeepers (ISCO code 201) is less obvious but could possibly also be explained by difference in equipment usage over time. The higher exposure that was found for motor vehicle drivers (ISCO code 633) in our study is very likely to be caused by the drivers in central parts of Stockholm in this study compared to the drivers in the countryside in the previous study, since there are many more sources of magnetic fields in a large city. In the Floderus JEM, cooks (ISCO code 912) and waiters (ISCO code 921) did not have very high levels ( 0.19 and $0.11 \mu \mathrm{T}$ respectively) but were based on few measurements and also included kitchen supervisors and kitchen maids. In our study these four occupations all had high average levels of exposure and were altogether represented by 32 measurements. Finally, we found a geometric mean of $0.19 \mu \mathrm{T}$ among attendants in psychiatric care (ISCO code 042); the Floderus estimate was only $0.08 \mu \mathrm{T}$. However, in the Floderus study this estimate also included subjects from registered nurses (ISCO code 040) and practical nurses (ISCO code 043), occupations in which we found low exposure levels.

An occupation that has been suggested as highly exposed is seamstresses (ISCO code 711), which was also included in this study. However, the seamstresses we measured were not working in the garment industry since these industries are very uncommon in Sweden today and have most likely never been a big employer of seamstresses in Stockholm County. The seamstresses in this study in general had their own small businesses and the intermediate level of exposure reported here is therefore not comparable to the very high levels reported elsewhere. ${ }^{16}{ }^{17}$

The comparison of exposure distribution in the female working population according to the census 1980 showed that about $16 \%$ of the women are highly exposed $(\geqslant 0.20 \mu \mathrm{T})$ but only $5 \%$ would be classified as such if the JEM for men was used. Furthermore, only $20 \%$ of the women with high exposure would be correctly classified as highly exposed by the JEM for men. Using the JEM for men in an epidemiological study would hence cause not only loss of power but could also dilute any effects through misclassification of the 
Table 3 Correlations between parameters (Pearson/Spearman) based on 471 subjects' measurements ( $\mu$ T) during the workday

\begin{tabular}{llllll}
\hline & Arithmetic mean & Geometric mean & Median & 90th centile & Max \\
\hline Geometric mean & $0.83 / 0.95$ & & & & \\
Median & $0.77 / 0.93$ & $0.97 / 0.98$ & & & \\
90th centile & $0.94 / 0.95$ & $0.66 / 0.88$ & $0.58 / 0.85$ & & \\
Max & $0.25 / 0.40$ & $0.15 / 0.29$ & $0.12 / 0.28$ & $0.21 / 0.39$ & $0.94 / 0.88$ \\
RC* & $0.38 / 0.61$ & $0.27 / 0.48$ & $0.23 / 0.46$ & $0.35 / 0.61$ & $0.25 / 0.32$ \\
RCs† & $-0.17 /-0.21$ & $-0.12 /-0.16$ & $-0.11 /-0.12$ & $-0.21 /-0.27$ & $0.27 / 0.29$ \\
\hline
\end{tabular}

*Rate of change.

†Standardised rate of change.

Table 4 Distribution of exposure in the population of women gainfully employed in Stockholm County 1980 by using JEM for women (ours) and JEM for men (Floderus et al, 1996)

\begin{tabular}{|c|c|c|}
\hline Geometric mean of TWA & JEM for women (\%) & JEM for men (\%) \\
\hline$\leqslant 0.10 \mu \mathrm{T}$ & 21.4 & 7.2 \\
\hline $0.11-0.20 \mu \mathrm{T}$ & 48.3 & 47.4 \\
\hline $0.21-0.30 \mu \mathrm{T}$ & 13.7 & 4.4 \\
\hline$>0.30 \mu \mathrm{T}$ & 3.0 & 1.0 \\
\hline Missing & 13.6 & 40.0 \\
\hline
\end{tabular}

exposure. Previous studies have identified occupations with high exposures to magnetic fields as occupations most commonly held by men. In this study we identified several occupations that are common among women and have high levels of magnetic fields. We also presented several different parameters of the exposure, making this JEM available for exposure assessment in future studies of magnetic fields in relation to various health outcomes among women.

\section{ACKNOWLEDGEMENTS}

The authors would like to thank Dr Birgitta Floderus and Lic Karin Stenlund for sharing their data and for helpful discussion of the results.

\section{Authors' affiliations}

U M Forssén, M Feychting, Institute of Environmental Medicine, Karolinska Institutet, Stockholm, Sweden

G Mezei, Electric Power Research Institute, Palo Alto, California, USA G Nise, Department of Public Health Science, Division of Occupational Health, Karolinska Institutet, Stockholm, Sweden

Funding: Electric Power Research Institute, California, Research and Development Agreement no. EP-P4618/C2247 and ELFORSK-Swedish Electrical Utilities R\&D company.

\section{REFERENCES}

1 IARC Working Group on the Evaluation of Carcinogenic Risks to Humans IARC monographs on the evaluation of carcinogenic risks to humans, vol. 80 . Lyon, France: IARC Press, 2002.

2 Li DK, Odouli R, Wi S, et al. A population-based prospective cohort study of personal exposure to magnetic fields during pregnancy and the risk of miscarriage. Epidemiology 2002;13:9-20.

3 Lee GM, Neutra RR, Hristova L, et al. A nested case-control study of residential and personal magnetic field measures and miscarriages. Epidemiology 2002;13:21-31.

4 McCurdy AL, Wijnberg L, Loomis D, et al. Exposure to extremely low frequency magnetic fields among working women and homemakers. Ann Occup Hyg 2001;45:643-50.

5 Deadman JE, Infante-Rivard C. Individual estimation of exposures to extremely low frequency magnetic fields in jobs commonly held by women. Am J Epidemiol 2002;155:368-78.

6 Burch JB, Reif JS, Yost MG, et al. Nocturnal excretion of a urinary melatonin metabolite among electric utility workers. Scand J Work Environ Health 1998;24:183-9.

7 Floderus B, Persson T, Stenlund C. Magnetic-field exposures in the workplace: reference distribution and exposures in occupational groups. Int $J$ Occup Environ Health 1996;2:226-38.

8 Feychting M, Forssen U, Floderus B. Occupational and residential magnetic field exposure and leukemia and central nervous system fumors. Epidemiology 1997:8:384-9.

9 Feychting M, Pedersen NL, Svedberg P, et al. Dementia and occupational exposure to magnetic fields. Scand J Work Environ Health 1998;24:46-53.

10 Feychting M, Floderus B, Ahlbom A. Parental occupational exposure to magnetic fields and childhood cance. Cancer Causes Control 2000;11:151-6.

11 Forssen UM, Feychting M, Rutqvist LE, et al. Occupational and residential magnetic field exposure and breast cancer in females. Epidemiology 2000;11:24-9.

12 Rothman KJ, Greenland S. Modern epidemiology, 2nd edn. Philadelphia, PA: Lippincott-Raven, 1998.

13 Forssen UM, Ahlbom A, Feychting M. Relative contribution of residential and occupational magnetic field exposure over twenty-four hours among people living close to and far from a power line. Bioelectromagnetics 2002;23:239-44.

14 Kelsh MA, Kheifets L, Smith R. The impact of work environment, utility, and sampling design on occupational magnetic field exposure summaries. AlHAJ 2000;61:174-82.

15 Harris C, Boivin W, Boyd S, et al. Electromagnetic field strength levels surrounding electronic article surveillance (EAS) systems. Health Phys 2000;78:21-7

16 Hansen NH, Sobel E, Davanipour Z, et al. EMF exposure assessment in the Finnish garment industry: evaluation of proposed EMF exposure metrics. Bioelectromagnetics 2000;21:57-67.

17 Kelsh MA, Bracken TD, Sahl JD, et al. Occupational magnetic field exposures of garment workers: results of personal and survey measurements. Bioelectromagnetics 2003;24:316-26. 